\title{
Stability of compacted rockfill slopes
}

\author{
J. A. CHARLES and M. M. SOARES (1984). Géotechnique 34, No. 1, 61-70
}

\section{Dr J. R. Greenwood Department of Transport, Bedford}

The Authors have made an interesting attempt to simplify analysis of rockfill slopes and to incorporate the possible curvature of the Mohr failure envelope under low stresses.

In considering the circular arc stability analysis (Fig. 1 of the Paper) the Authors fall into the fundamental trap (as others have done before) of resolving total stresses rather than effective stresses to find the normal effective stress acting on the base of the slice.

The effective stress on the base of the slice should be given as

$$
\sigma^{\prime}=\frac{(W-u t) \cos \alpha}{l}
$$

and not

$$
\sigma^{\prime}=\frac{W \cos \alpha-u l}{l}
$$

as given in equations (6) and (8) of the Paper.

With this simple correction the basic Fellenius or Swedish method becomes readily applicable to all stability analyses and regardless of whether or not porewater pressures are present credible results are obtained in all cases (Greenwood, 1983).

Using equation (1) the original equation (9) now becomes

$$
F=\frac{1}{\sum W \sin \alpha} \sum A[(W-u t) \cos \alpha]^{b} l^{1-b}
$$

where the slice width $t$ is approximately equal to $l \cos \alpha$.

Furthermore if the factor of safety is defined as suggested by Lambe \& Whitman (1969) as the shear resistance along the slip surface divided by the shear stress along that surface then equation (3) can also be used for the approximate analysis of non-circular slip surfaces.

The difficulties encountered by the Authors when analysing steep slopes $(\cot \beta<0.5)$ are, I believe, a result of the incorrect resolution of total stresses in the Fellenius and Bishop methods. Steep slope angles $\beta$ would have correspondingly steeper angles $\alpha$ at the base of each slice in the stability analysis. It can be seen from equation (2) that $\sigma^{\prime}$ becomes unrealistically negative when $\alpha$ is large whereas in equation (1) $\sigma^{\prime}$ would always remain positive, (unless pore pressures exceeded the total weight of the soil, i.e. 'quick' conditions). Greenwood (1983) demonstrated that the Bishop solution gives unrealistically high factors of safety for deep slip circles whereas the unmodified Fellenius solution gives unrealistically low values.

In conclusion the limitations of any stability charts must be recognized in that varied geometry, surcharge loading, layered soils and varying water pressures cannot easily be taken into account. The design engineer therefore requires a simple method of analysis to apply to his own particular problem.

The Bishop solution may be more rigorous and may give a slightly higher factor of safety in certain cases but, as the Authors point out, its mathematical stability is sometimes in question. The difference between solutions is small compared with the uncertainty in determining appropriate parameters for the fill and I consider that the readily applied modified equation (3) is adequate for routine analyses of rockfills. It gives results identical with the Authors' solution $\left(F_{F}\right)$ when porewater pressures are zero and more credible results when water pressures are present. It has the advantage over the Bishop equation in that it does not require iteration or initial assumptions and it gives sensible consistent results for all failure surfaces whether shallow or deep.

\section{REFERENCES}

Greenwood, J. R. (1983). A simple approach to slope stability. Ground Engng 16, No. 4, 45-48.

Lambe, T. W. \& Whitman, R. V. (1969). Soil mechanics, pp. 363-365. New York: Wiley.

\section{Authors' reply}

In following the Fellenius and Bishop methods of circular arc stability analysis, Greenwood believes that we have fallen into the fundamental trap of resolving total stresses rather than effective stresses to find the normal effective stress acting on the base of the slice'. In fact the normal effective stress was determined by resolving forces. Fig. 1 illustrates the stability 


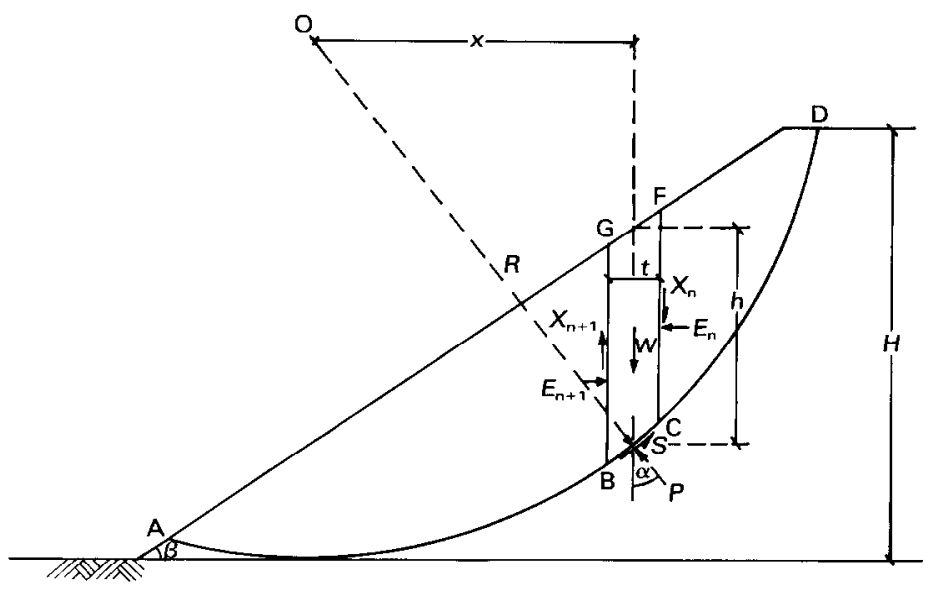

Fig. 1

analysis used in the Paper. From a consideration of the equilibrium of the slice BCFG and resolving forces normal to the slip surface we obtain

$$
P=\left(W+X_{n}-X_{n+1}\right) \cos \alpha-\left(E_{n}-E_{n+1}\right) \sin \alpha
$$

In the Fellenius method interslice forces are ignored, i.e. it is assumed that

$$
\left(X_{n}-X_{n+1}\right) \cos \alpha-\left(E_{n}-E_{n+1}\right) \sin \alpha=0
$$

and therefore equation (4) reduces to

$$
P=W \cos \alpha
$$

The normal effective stress acting on the base of the slice

$$
\sigma^{\prime}=\frac{P}{l}-u
$$

where $u$ is the porewater pressure at the slice base and $l$ is the length of the base of the slice.

Combining equations (6) and (7) we obtain

$$
\sigma^{\prime}=\frac{W \cos \alpha}{l}-u
$$

Equation (8), which Greenwood considers incorrect, is thus seen to be derived from the definition of normal effective stress in equation (7) and the Fellenius assumption about interslice forces on which equation (6) is based. Equation (8) is an approximation whose usefulness depends on the extent of the error involved in the Fellenius method of stability analysis. Bishop developed a more rigorous method of analysis in which interslice forces were not ignored. In his semirigorous analysis it is assumed that the resultant of the interslice forces acts horizontally, i.e.

$$
X_{n}-X_{n+1}=0
$$

This assumption leads to a relationship for $\boldsymbol{\sigma}^{\prime}$ that is different from that given in equation (8).

It can be shown that the expression

$$
\sigma^{\prime}=\frac{W \cos \alpha}{l}-u \cos ^{2} \alpha
$$

which Greenwood had derived by 'resolving effective stresses', corresponds to an assumption about interslice forces such that

$$
\left(X_{n}-X_{n+1}\right) \cos \alpha-\left(E_{n}-E_{n+1}\right) \sin \alpha=u l \sin ^{2} \alpha
$$

and on this assumption

$$
P=W \cos \alpha\left(1+r_{u} \tan ^{2} \alpha\right)
$$

Equations (11) and (12) should be compared with the corresponding equations (5) and (6) in the Fellenius method. It is easier to understand the physical significance of the assumptions about interslice forces that are made in the Fellenius and Bishop semirigorous stability analyses than it is to understand the significance of the assumption about interslice forces that underlies Greenwood's expression for $\sigma^{\prime}$ given in equation (10).

It should be noted that, although the stability analysis equations were developed in the Paper with a pore pressure term in them, the stability charts are for rockfill and all the charts refer to the case where $u=0$. Therefore difficulties that were encountered with very steep slopes and high values of shear strength parameter $b$ could not have been connected with the different expressions for $\sigma^{\prime}$ in equations (8) and (10), as these two expressions are identical when $u=0$. Where there are pore pressures in a slopc, the difference might be of importance.

The comment on the limitations of stability charts in practical situations is fully accepted. 